Voix et Images

\title{
Des romans pour pas cher : la littérature de masse, sous le scalpel des universitaires
}

\author{
Joseph Bonenfant, André Brochu et Gilles Marcotte
}

Volume 10, numéro 1, automne 1984

Littérature canadienne-anglaise

URI : https://id.erudit.org/iderudit/200463ar

DOI : https://doi.org/10.7202/200463ar

Aller au sommaire du numéro

Éditeur(s)

Université du Québec à Montréal

ISSN

0318-9201 (imprimé)

1705-933X (numérique)

Découvrir la revue

Citer ce document

Bonenfant, J., Brochu, A. \& Marcotte, G. (1984). Des romans pour pas cher : la littérature de masse, sous le scalpel des universitaires. Voix et Images, 10(1),

131-139. https://doi.org/10.7202/200463ar d'utilisation que vous pouvez consulter en ligne. 


\title{
Des romans pour pas cher: la littérature de masse, sous le scalpel des universitaires
}

\author{
par Joseph Bonenfant, André Brochú et Gilles Marcotte
}

\section{Commentaire de Joseph Bonenfant, Université de Sherbrooke}

Commençons par les extrêmes. Quand on lit Proust, l'histoire a déjà eu lieu. Le récit donne forme à une vie déjà vécue. On prête forcémént attention à l'écriture, acte d'appropriation du passé et lieu d'élaboration du discours. Le suspense est attaché à l'écriture elle-même, êt on lit trop vite.

Dans les petits romans que j'ai lus, c'est le contraire. On oublie ce qui se passe dans l'ècriture pour entrer dans une histoire qui se fabrique sous nos yeux. On subit le feu de l'action dans le présent, on en anticipe les rebondissements, on est sans cesse projeté en avant. Bref, on ne lit pas assèz vite.

C'est une lecture truffée de big bangs, où alternent, chez le lecteur, les ah non! et les ouf! On n'a pas le temps de se remettre d'une problématique vite réglée, ou escamotée, qu'on est précipité dans un mensonge tactique; on voit des morts qui parlent, on entênd des cris de désespoir; on est bousculé par des scénarios très ou trop mobiles, affolé ici et là par quelques gouttes de sang. Qu'est-ce qu'une lecture aussi captivante capte de moi? Est-ce ma liberté de rêver? Ou de musarder sur le texte? À quoi suis-je contraint?

Chaque roman me propose en clair un message, ou m'ouvre une piste. Dans l'Amnésique, de Pierre Saurel, je suis stupéfait d'entendre Brien, le détective qui aide (le mot revient dix fóis), qui fait du bien à tout le monde, proclamer : "Il faut faire face à la réalité » (p. 16). Dans la Vengancé des Mohawks, c'est Vaughn, encerclé et soumis, qui déclare : «Mourir quánd on est riche, c'est triste" (p. 28). Dans la Clique des femmes mariées, c'ést avec émotion que j'entends Gisèle, la révoltée, lancer, au móment d'être assassinée par le souriant Mack : «C'est mon corps et je crois avoir le droit de faire ce que je désire avec» (p. 27). Ou encore Huguette, 29 ans; au moment d'être noyée dans sa baignoire par le même sinistre Mack souriant: «Je suis trop jeune pour mourir... Ayez pitié, ce n'est pas humain» (p. 31-32). 
Qu'est-ce que je fais de ces messages? J'en pleure ou j'en ris? Dans l'indécision, je m'en méfie plutôt. Je prends la distance que je peux. Bref, je résiste à mes émotions, et c'est d'autant plus facile que je ne suis pas ému ; je ne peux avoir cette naïveté. Suis-je un mauvais lecteur? Jusqu'ici, oui.

Ce qui m'intéresse dans ces romans, ce sont les messages involontaires, les clichés idéologiques, les passions humaines nues, les prises et les crises de positions sociales. Par exemple, j'aime bien Ravena la meneuse, sa cupidité de chercheuse d'or et de violeuse du sanctuaire sacré des Catacombes des grands chefs Mohawks. Elle dirige la clique des forbans, Runt, Ken et Vaughn, jusqu'à lả mort. J'aime bien Louise, dans l'Amnésique, calculatrice sardonique, meurtrière à froid pour s'enrichir avec les assurances, séductrice désespérée. Dans la Clique des femmes mariées, j'aime bien Martha, qui tient tête, au prix de sa.vie, à l'infâme Monsieur Mystère, et Gisèle, et Madame Dumoulin, et Huguette qui, elles aussi, meurent martyres de leur érotisme putassier.

Ailleurs, on rencontre, à l'état pur, des gens perfides, ou infâmes, ou impitoyables. On se croirait dans Homère, sauf que par-dessus les siècles, dans la dérive des genres et selon les variations de la demande, la poésie a foutu le camp. On remarque l'absence cruelle de nature animale et végétale. On est dans un désert d'humanité pure, dans un champ clos où s'affrontent le bien et le mal, et il faut bien le dire, dans les trois spécimens examinés, la femme et le mâle.

Je viens d'effleurer des contenus. Mais, pour ma part, je crois que le plus intéressant à étudier dans ces romans populaires, ce serait les phénomènes stylistiques, les clichés rhétoriques, les stéréotypes culturels, les sémiosis mythiques, sociales, politiques:

a) Albert Brien, détective national des Canadiens français;

b) Pịt Verchères, les exploits légendaires du capitaine fédéral, le roi de l'Ouest canadien;

c) des référents institutionnels: l'Amnésique: grand roman policier de Pierre Saurel; la Clique..., grand roman d'aventures.

On remarquerait aussi des stratégies discursives, malgré une linéarité plate et une prédominance frappante des prolepses sur les analepses, des agrafes narratives passe-partout, dans la Vengeance des Mohawks, du genre : «à ce moment précis»; "et lentement», "trois mois plus tard»; dans la Clique, les menaces, parfaitement homériques, qui précèdent les actes : «Je ne veux pas que tu quittes cette terre sans souffrir un peu» (p. 17); «Madame, si je peux oser vous appeler ainsi, je suis ici pour vous tuer» (p. 26); de nouveau dans la Vengeance des Mohawks, le phénomène régulier de la coordination pour annoncer la mort: 
p. 24 : «l'Indien poussa un terrible cri

et s'écroula lourdement dans les broussailles»;

p. 25: «Runt tournoya sur lui-même et s'abattit lourdement sur le sol»;

p. 29: «Ken poussa un long cri et culbuta dans la poussière»;

p. 31: «Ravenna eut un sursaut et elle glissa sur le sol mou».

Finalement, quand nous lisons ces romans populaires, vite écrits et vite lus - la qualité de la langue ne laisse-t-elle pas parfois à désirer, les coquilles ne reflètent-elles pas un travail à la vapeur? - que cherchons-nous? Est-ce le simple plaisir de la lecture, comme leurs premiers et bien identifiables destinaires? Qu'avons-nous à faire dans cette galère de la littérature de masse, nous gens savants qui participons activement à un congrès pour l'avancement de la science, et qui avons fait des thèses de doctorat sur des écrivains d'autres pays et d'autres siècles?

Devons-nous ausculter, examiner, palper cette littérature sans prétention, cette littérature à succès, avec les hantises, et les instruments, du sociologue, de l'historien, du psychocritique; du psychanalyste, du thématicien, du sémioticien; du narratologue? Oui, sans doute. Oui, certainement. Et je souhaite qu'on le fasse sans bouder son plaisir. Comme quand on lit Proust. Ou Astérix.

P.-S.: Après les discussions qui ont eu lieu lors de cet atelier consacré à la littérature de masse, je dois ajouter que je ne me suis jamais senti honteux de ma préférence pour la littérature d'élite, que connaît et pratique tout bon lecteur de la revue Voix et Images. Je n'ai pas non plus le sentiment d'avoir, par ce texte, exprimé «avec un raffinement distingué, mon horreur» pour cette littérature de masse.

On peut se demander pourquoi il n'y aurait pas, dans le ciel littéraire, plusieurs demeures, comme il y en a dans le ciel des gastronomes, ou des téléspectateurs. Pourquoi ne parle-t-on jamaìs de gastronomie d'élite? Comme la littérature de masse, la littérature d'élite correspond à des intérêts, à des moyens et à des projets de çlasse. Quelle honte à l'affirmer ? On a du pouvoir, ou on n'en a pas. À forcè d'analyser nos goûts et de les défendre, à force de nous salir les mains au contact des «basses ouvres i» de la littérature, n'allons-nous pas, nous distingués universitaires, devenir méconnaissables? La mauvaise conscience va finir par nous tuer. 


\section{Commentaire d'André Brochu, Université de Montréal}

Invité à commenter six échantillons de littérature dite "de masse», il me faut dire d'abord que, à mon avis, la littérature de masse, ce n'est pas uniquement ce genre de romans populaires qui a fleuri après la guerre. C'est aussi, plus près de nous, le roman fantasitique, le roman de science-fiction et d'autres formes de paralittérature qui, tout en visant un vaste public, ont parfois des critères très élevés de qualité. Mais notre littérature de masse; c'est peut-être d'abord et surtout cette immense production orale, celle des contes et légendes, qui a constitué la littérature du peuple avant que l'alphabétisation se généralise (et elle ne s'est pas généralisée avant le dix-neuvième siècle, et souvent le vingtième). Ixe-13 est un phénomène récent parce qu'il est impossible dans une société peu alphabétisée et peu urbanisée. Nous sommes encore tout près de la société et de la littérature orales (et paysannes), et c'est depuis peu que notre littérature de masse a pris un caractère différent : elle est dorénavant écrite et véhicule un imaginaire le plus souvent urbain.

Entre la littérature de masse d'autrefois (la littérature orale) et ce qu'on appelle la "grande » littérature, il n'y avait pas solution de continuité. $\mathrm{Au}$ contraire, de tous temps les écrivains se sont alimentés aux sources populaires, et ils le font encore aujourd'hui. Les contes et légendes ont été recueillis par les ethnologues et les folkloristes et forment un corpus, inçomplet sans doute mais précieux, qui a les caractères et la dignité d'une littérature. Ce qui la distingue le plus fondamentalement, c'est que les énonciateurs en sont les gens du peuple eux-mêmes.

Le roman populaire du genre Ixe-13 (ou M. Mystère, ou Pit Verchères...) me semble bien différent de ceux que je viens d'évoquer. Il s'agit de littérature de masse, sans doute, mais pour éviter toute confusion, sinon toute polémique, parlons de littérature de masse niaiseuse ou, pour prêter moins à contre-sens, de littérature niaiseuse pour les masses. Niaiseux veut dire niais, en plus familial.

Je donne de petits exemples.

Dans Monsieur Mystère (le roman le plus inepte et le plus mal écrit), la pauvre Martha est condamnée à être dévorée par les fourmis. On lit ceci : «Une demi-heure plus tard,' il ne reste plus que quelques membres à Martha qui n'ont pas encore été rongés par les fourmis.» Quelques membres! Martha était-elle un mille-pattes? Quelques membres, cela veut dire qu'il lui en reste au moins quatre et qu'elle n'a pas été dévorée du tout. Eh bien, la voilà la niaiserie. L'inconscient bonasse vole au secours de la victime, l'auteur est incapable d'être méchant.

Albert Brien non plus, qui dit à l'amnésique qui a fait appel à ses services : «Écoutez, Limoges, je suis prêt à croire que vous n'avez pas tué l'époux de votre femme.» Il est bon de se souvenir ici que Limoges passait pour mort et que sa femr le s'était remariée. La formule n'en reste pas moins cocasse et, là encore, elle sert à escamoter la violence. 
Un autre exemple, beaucoup plus léger, dans Ixe-13. Le méchant capitaine soviétique, gardé à vue par la sympathique Gaby, réussit à se défaire de ses liens. Il cherche alors à détourner l'attention de sa gardienne:

Gaby se retourna l'espace d'une seconde seulement.

Comme s'il avait eu un ressort quelque part, le capitaine bondit de sa chaise.

Je vous laisse à deviner où le capitaine avait son ressort.

Alors que, dans les exemples précédents, l'inconscient gommait le sang, ici il se fait plus gaulois et y va d'un peu de cul. L'auteur n'a sans doute pas le temps de s'en apercevoir, et toutes les conditions se trouvent réunies pour ce que j'appellerai un effet de niaiserie.

Dernier exemple, qui se passe de commentaire. C'est tiré de la Fille $\grave{a} /$ de personne (à en page couverture, de à l'intérieur) : «Laurie avait pris la pierre scintillante entre ses doigts jaunis par l'alcool.»

Face à la littérature de masse niaiseuse, ma position est la suivante.

Rien n'est sans intérêt, mais il faut savoir où se situe l'intérêt.

Les romans populaires publiés au Québec après la dernière guerre sont certainement intéressants pour les sociologues et les psychologues; mais c'est à eux de dire en quoi ils le sont. Et je leur pose une question: une grande œuvre littéraire est sans doute moins immédiatement le reflet de la société ou de l'inconscient que ne l'est le roman populaire; pourtant n'y a-til pas plus à en tirer, l'œuvre étant incomparablement plus riche?

Je poserais la même question aux sémioticiens ou aux narratologues. Les lois du discours narratif sont plus évidentes dans un roman constitué de recettes très simples. Mais le Rouge et le Noir n'apprend-il pas davantage sur le discours narratif que James Bond?

Mais même si le sociologue, le psychologue, le sémioticien font leur profit de la lecture d'Ixe 13, le littéraire, lui, ne voit dans ces textes que niaiserie, facilité, procédés sommaires, sémantique primaire.

Il pourrait cependant arriver qu'un roman destiné au grand public soit un chef-d'œuvre et rejoigne dès lors les rangs des ouvres littéraires au sens strict. Quelques-uns de nos romanciers, présents et passés, se sont d'ailleurs approchés de certaines formes de littérature populaire. Je pense à Roger Lemelin, à Yves Beauchemin, à Gabrielle Roy (Guy Laflèche, on le sait, qualifie Bonheur d'occasion de roman populaire). Si la littérature de masse, c'est le Matou, je veux bien qu'elle existe, et j'en redemande! Si c'est Pierre Saurel, je la laisse à ceux que cela intéresse. Je ne méprise pas Pierre Saurel, c'est-à-dire Pierre Daignault, qui du reste ne cherche pas à être lu des intellectuels. Je dis seulement que cette littérature de pur divertissement, de pure évasion, eșt le contraire de la vraie littérature. 
Étant bien entendu que je ne m'y intéresse pas, je me demande cependant si elles ne devrait pas intéresser une autre catégorie de paralittéraires, ceux qui s'occupent de la littérature de jeunesse. Je me demande dans quelle mesure Ixe 13, Pit Verchères, Albert Brien, ne sont pas les héros de la véritable littérature de jeunesse d'une certaineépoque. Jeune, je lisais leurs aventures de préférence à beaucoup d'autres.

Aucune barrière de classe, par conséquent, ne m'en empêchait. Je me pose la question : est-ce une barrière de classe qui, aujourd'hui, m'empêche de goûter ce que je goûtais jadis? Il faudrait conclure alors que l'enfant, de même qu'il est, selon Freud, un pervers polymorphe, serait une sorte de social polymorphe... Pourtant il n'en était rien: j'étais bien moins conscient de mes préjugés de classe, pourtant énormes, quand j'étais enfant, que je ne le suis maintenant. Alors, que s'est-il passé?

Il s'est passé que la littérature s'est mise à signifier, pour moi, autre chose que l'évasion. Une sorte d'aventure du langage et de l'exister, tout ensemble.

Quand je lis de la vraie littérature, je suis à la fois plus pris par le texte et plus libre devant lui, que lorsque je lis Ixe 13 ou Monsieur Mystère. Plus pris parce que le vrai texte littéraire me dépasse, comme il dépasse sans doute l'auteur lui-même, pour la bonne raison que la grande littérature est dépassement, invention toujours à nouveaux frais de la totalité du langage et du vécu. Et ce dépassement m'est communiqué de façon plurielle : le texte met en éveil toutes mes facultés de sentir et de penser.

Le roman de masse est, lui, un roman spécialisé. Il ne présente que des moignons de vérité humaine, jamais l'être au complet. Il flatte en moi l'ignoble détective, l'abominable shérif, quand ce n'est le débile $M$. Mystère. Il ne fait appel ni à mon intelligence ni à ma sensibilité, seulement à mon goût pour les sensations fortes. Voilà du reste pourquoi il me piège, me prive de ma liberté de lecteur. Il me coince dans des situations invraisemblables où, pour que le héros auquel je m'identifie puisse échapper à la mort, je dois consentir à des péripéties qui font affront à l'intelligence. Je cite ici la fin de la Vengeance des Mohawks:

Mais Pit Verchères poussant un cri vengeur s'était précipitamment levé derrière son abri et lança sa carabine vide à toute volée en direction de la jeune femme.

Vive comme l'éclair elle releva le canon de sa winchester et fit feu...

La balle ricocha contre le fer bleu de la carabine au vol et vrillant dans une plainte prolongée, le plomb revint sur sa course et frappa Ravenna en pleine poitrine.

Demander au lecteur de gober un tel merveilleux, c'est peut-être là en fait que se trouve le mépris. 


\section{Commentaire de Gilles Marcotte, Université de Montréal}

La première question que je me suis posée, après avoir lu la Véngeance des Mohawks, dans la série des Exploits du légendaire Pit Verchères, le Roi de l'Ouest canadien, la Belle Mathilde, dans la série Mon roman d'amour, la Clique des femmes mariées, dans la série des Exploits fantastiques de Monsieur Mystère, l'Amnésique, dans Les Aventures policières d'Albert Brien, détective national des Canadiens français, l'Imposteur, dans les Aventures étranges de l'agent IXE-13, et la Fille à personne, dans la série Diane la belle aventurière, - la première quéstion que je me suis posée, donc, est la suivante : pourquoi diable $m$ 'a-t-on envoyé ces petits romans, à moi, professeur titulaire à l'Université de Montréal, pourvu d'une réputation de sérieux assez étanche merci?

C'était, peut-être, pour me distraire de mes travaux ardus? Mais il se trouve que, si la pornographie ne m'attire pas et si le western ne m'intéresse qu'au cinéma, en revanche je suis un assez grand consommateur de romans policiers et de romans d'espionnage. Et la première chose, la toute première chose qu'il faut dire des brochures publiées par les éditions Police-Journal, me semble-t-il, c'est qu'elles sont d'une pauvreté, et j'oserais dire d'une débilité tout à fait consternantes. Je sais à quoi je m'expose en faisant une telle déclaration : à me faire traiter d'élitiste, et c'est bien ce qui peut arriver de pire à un intellectuel, à un universitaire québécois. Mais, en tant qu'amateur - et non professeur ou critique, cela viendra peut-être plus tard - , je ne puis reconnaître à ces petits romans qu'une seule qualité, la brièveté : on ne s'ennuie pas trop longtemps. Et puis parfois, oui, on leur doit quelques éclats de rire. Lorsque, par exemple, à la fin de La Vengance des Mohawaks, la belle et méchante Ravenna se trouve avec une carabine chargée dans les mains devant Pit Verchères qui, lui, n'a plus de balles. Pit lance sa carabine à la tête de Ravenna qui tire aussitôt, mais la balle - je cite «ricocha contre le fer bleu de la carabine au vol et vrillant dans une plainte prolongée, le plomb revint sur sa course et frappa Ravenna en pleine poitrine». Faut le faire!... Le romancier ajoute, à propos de la même Ravenna: «Elle eut un sursaut»... Le lecteur aussi !

Mais voilà, ces petits romans - qui ont été publiés, me dit-on, entre 1945 et 1967 - ont été lus, à l'époque, par un grand nombre de gens. Qui étaient-ils? Je connais ceux qui ne les lisaient pas, ou guère : ceux qui vivaient autour de moi, dans mon milieu très-petit-bourgeois, minuscules bourgeois. Les lecteurs de romans populaires, pourtant, ne devaient pas être loin, et ils étaient sans doute à peine plus pauvres que moi. Je les imagine comme des personnages de Marcel Dubé ou mieux de Michel Tremblay, trouvant dans ces brochures leur pitance de rêve, d'évasion, la version québécoise de ce qu'ils voyaient par ailleurs dans les films hollywoodiens de série $B$. Je connais assez bien les films de série B, qui sont des chefs-d'œuvre de subtilité auprès des romans de Police-Journal; mais sans connaître les 
romans populaires qui se consommaient à l'époque dans d'autres pays les Etats-Unis, l'Italie ou la France par exemple -, je soupçonne qu'ils étaient moins démunis que les nôtres. Notre production, dans ce domaine, n'est-elle pas un autre témoignage d'une pauvreté - économique et culturelle, indissolublement - que nous n'avons certes pas fini de mesurer? On remarquera que, dans chacun de ces cinq romans, le système de compensation joue avec une brutalité, une naïveté tout à fait extraordinaires. La présence française est presque disparue dans l'Ouest canadien ? Qu'à cela ne tienne, le légendaire Pit Verchères rétablit à lui seul la situation. Vous croyez que nous ne comptions pour rien sur la scène internationale? C'est que vous ne connaissez pas IXE-13, l'espion des espions. Nous voici même champions du vice - ce n'était pourtant pas notre spécialité ! - avec Monsieur Mystère, l'Homme au cerveau diabolique. Il n'est pas jusqu'à la très belle Mathilde, l'étudiante fatiguée d'être belle et d'être poursuivie pour sa beauté, qui ne puisse être considérée comme une sorte de revanche du sort sur un destin national trop gris... Tout cela fonctionne de façon mécanique, et sans que des effets un peu surprenants, des habiletés un peu spéciales, viennent rompre la monotonie du processus.

La deuxième question que je me suis posée est celle-ci : quel rapport ces textes ont-ils avec la littérature - celle qui se produisait durant la même époque (1945 à 1967) au Québec, et celle d'aujourd'hui? La distance, entre les deux sortes de production - car il s'agit bien de deux sortes de production - est presque infinie. On ne vise pas le même public. Et on n'utilise pas les mêmes moyens. Les romans de Police-Journal n'appartiennent pas à ce qu'on a convenu d'appeler la littérature. Mais qu'est-ce donc que la littérature, quelle définition en donne-t-on, pour être autorisé à en exclure des textes qui ne semblent se distinguer en rien, formellement, des récits littéraires? Pour nous éclairer un peu là-dessus, pensons au roman d'Yves Beauchemin, le Matou. Voici un roman d'action, vif, sans subtilité psychologique, qui semble tirer son extraordinaire vitalité d'une reprise de contact avec... le roman populaire, justement, selon un processus que Wellek et Warren appellent la « rebarbarisation » de la littérature - et sans lequel la littérature, à certaines époques, risquerait de se perdre dans l'abstrait, l'exsangue. Mais il est évident que le roman de Beauchemin, à partir de formules de récit semblables à celles d'un Pierre Saurel ou d'un Maurice Lenoir, s'emploie à fabriquer de la surdétermination sémantique, et par là s'insère dans un ensemble intertextuel radicalement différent. Ce n'est pas par hasard - ou uniquement pour des causes étroitement sociologiques - que la critique littéraire a ignoré les romans de Pierre Saurel, et parlé de celui de Beauchemin; c'est que celui-ci, par la forme de langage même qu'il pratique, appelle le commentaire, et que l'autre ne l'appelle pas. Le roman populaire ne fait pas parler, il ne parle pas; et nous lui faisons peut-être violence aujourd'hui, la plus grande violence, en le convoquant dans notre assemblée parlante, notre très littéraire assemblée. 
D'où, justement, la troisième question : que viennent faire ces romans populaires dans un colloque organisé, animé par des professeurs de lettres? Eh bien, comme chacun ne l'ignore pas, c'est qu'ils sont entrés dans une classe d'objets d'études spécialement créée à leur intention, qui s'appelle la "para-littérature", et sur laquelle un certain nombre d'ouvrages très savants ont déjà été écrits. Du point de vue institutionnel - qui invite toujours à quelque cynisme, on m'excusera - , l'annexion de ces nouveaux territoires constitue pour les professeurs de lettres un double profit:

1) D'abord, une extension considérable du champ, du domaine, particulièrement bienvenue à une époque où la concurrence est de plus en plus vive, entre les professeurs de lettres, dans la poursuite des biens symboliques (qui ne sont pas toujours uniquement symboliques, comme chacun sait). L'exploration extrêmement détaillée du champ littéraire québécois, de même que son utilisation dans un nombre toujours plus grand de pays étrangers, relèvent me semble-t-il du même désir, du même besoin.

2) D'autre part, l'entrée du roman populaire - en quel sens, populaire? Je laisse la question en suspens - permet aux études littéraires de se dédouaner pour ainsi dire, à une époque où plus qu'à d'autres peut-être, elles sont victimes d'une certaine suspicion, souvent partagée par les professeurs eux-mêmes. Quand on vit depuis quelques années dans une université, à l'abri des mouvements de foule, à étudier des textes difficiles dont souvent la consommation est assez réduite, il arrive qu'on se sente coupé de la vie, de la vraie vie qui est en bas, dans la rue, qu'on se sente péniblement inutile. Alors, on quitte Robbe-Grillet pour Maurice Lenoir, Jean Vernois ou Pierre Saurel. La littérature populaire, ça sent bon le peuple, la réalité réelle, la vie vivante. Quand je lis Stendhal ou Hubert Aquin, je fais partie des «happy few" (qui sont quand même devenus assez nombreux, avec le temps); quand je me plonge dans la lecture de la Clique des femmes mariées, je prends un bain de foule, je suis au restaurant du coin avec les filles et les gars, je suis l'un d'eux et l'un d'elles, pour un peu je cesserais d'être un intellectuel. Et je commence à lire, comme tout le monde :

Un soleil ardent fait briller le sable sur une plage de Floride.

Les vagues viennent caresser le sable chaud. Quelques mouettes lancent un cri de détresse.

Â l'horizon, un bateau se dessine.

En courant, Monsieur Mystère sort de l'eau.

Et cetera... 